\title{
Knowledge, Attitudes, and Practices on COVID-19 in Kurdistan Region of Iraq: An Online Cross-Sectional Survey
}

\author{
Nazdar Qudrat Abas ${ }^{1 *}$, Muhammad Amen ${ }^{2}$, Nazanin Abbas $^{3}$, Muhamad Yusif $^{4}$, Dilshad Jaff ${ }^{5}$ \\ ${ }^{1}$ Department of Psychology, College of Education, University of Garmian, Kalar, Kurdistan Region, Iraq \\ ${ }^{2}$ Department of Adult Nursing, College of Nursing. University of Sulaimani, Sulaimani, Kurdistan Region, Iraq \\ ${ }^{3}$ Kalar Private Technical Institute, Kalar, Kurdistan Region, Iraq \\ ${ }^{4}$ Research Center, University of Garmian, Kalar, Kurdistan Region, Iraq \\ ${ }^{5}$ Department of Maternal \& Child Health, Gillings School of Global Public Health, University of North Carolina at Chapel Hill, USA
}

Received 04 June 2021; revised 14 September 2021;

accepted 07 November 2021; available online 27 November 2021

doi: $10.24271 /$ psr.36

\begin{abstract}
Background: First COVID-19 case was recorded on February $\left(26^{\text {th }}, 2020\right)$ in Kurdistan Region of Iraq (KRI). Shortly after that number of cases increased significantly but, limited research has been conducted to assess the knowledge, attitudes, and practices of the population in the region about the pandemic.

Objectives: The objective of this study was to gain a better understanding of the people's awareness and their attitude towards the pandemic by conducting a knowledge, attitude and practice (KAP) survey.

Materials and Methods: A quantitative, descriptive, cross-sectional online survey, and non-probability, convenient sampling of 325 adults, was conducted during a complete lockdown period from (5th to 20th of April, 2020) in KRI.

Results: High levels of knowledge and practice, with divided positive and negative attitudes, were found among participants. The study found a positive relationship between knowledge and attitudes and the resulting practice.

Conclusions: Studies about the COVID-19 pandemic are very scarce in the KRI. There were no significance differences between the socio-demographic characteristics and levels of knowledge, attitudes and practices. We encourage further KAP studies in the KRI regarding COVID-19 for setting health awareness campaigns in promoting practical information on preventive measures to stop the spread of the virus accordingly.
\end{abstract}

\section{(C) 2022 Production by the University of Garmian. This is an open access article under the LICENSE}

https://creativecommons.org/licenses/by-nc/4.0/

Keywords: Attitudes, COVID-19, Garmian Region, Knowledge, Practice, Preventive Measures.

\section{Introduction}

In December (2019), coronavirus disease (COVID-19) has been first reported in Wuhan, China and began spreading rapidly through several countries ${ }^{[1]}$. On $11^{\text {th }}$ of March 2020, the World Health Organization (WHO) declared COVID-19 a pandemic ${ }^{[2]}$. By mid-August 2020, total confirmed cases surpassed 21 million globally. In the Kurdistan Region of Iraq (KRI), the first diagnosis of COVID-19 cases has been in February $26^{\text {th }} 2020$. By March $1^{\text {st }} 2021$ there have been 108,954 confirmed cases (1.8\%), with 103,514 (95\%) recoveries and 3,519 (3.2\%) fatalities out of a population over six million ${ }^{[3]}$.

* Corresponding author

E-mail address: nazdar.qudrat@garmian.edu.krd (Instructor).

Peer-reviewed under the responsibility of the University of Garmian.
Infection control measures are always recommended in preventing transmission of infection in infected settings ${ }^{[4]}$. Good knowledge is crucial for controlling infection spreads by enhancing correct practices in preventing measures, and hence to ensure better safety ${ }^{[5]}$. It has been proved that the best measures for infection control are good knowledge, positive attitudes, and healthy practice6. Knowledge about COVID-19 were provided widely, and from many resources, including international resources and local resources, Ministry of Health in Kurdistan Region of Iraq (KRI) has been providing knowledge and updates about COVID-19 through a special dashboard ${ }^{[3]}$.

Since the appearance of the pandemic, a number of knowledge, attitude and practice (KAP) studies have been carried out in Iraq and various regions of the KRI. Based on our knowledge, there have been three KAP studies conducted on the general population in Iraq ${ }^{[7,8,9]}$, and one study on the population residing in the KRI 
${ }^{[10]}$. Other similar studies in Iraq and KRI were mainly conducted among healthcare workers ${ }^{[11,12,13]}$.

KAP studies were carried out in Saudi-Arabia, Malaysia, China, and Nigeria [14, 15, 16, 17]. All studies found high levels of knowledge, positive attitudes, and safe practices. Some of the other KAP studies surveyed different sample groups such as medical students in Jorden ${ }^{[18]}$ and in Iran ${ }^{[19]}$, dentists in Jorden ${ }^{[20]}$, and health workers in China ${ }^{[21]}$ and Italy ${ }^{[22]}$.

In response to the pandemic, countries issued restrictive measures including total or partial lockdowns and travel restrictions to prevent the spread of the virus ${ }^{[23]}$. The same actions were implemented in the KRI on March $17^{\text {th }} 2020$, mainly in Garmian Region, which borders Iran where cases were surging. When the first case was detected in KRI, local authorities declared complete lockdown which included closing schools and universities and restricting movements, and social gatherings. Despite implementing these measures, cases started to raise steadily in all the four governorates of Kurdistan, including Garmian Region, especially after easing the first lockdown ${ }^{[3]}$.

The first case of Covid-19 was diagnosed on May 21 ${ }^{\text {st }}, 2020$ and shortly thereafter, the number of cases increased significantly, according to local health authorities (DoH Garmian, February $\left.22^{\text {nd }}, 2021\right)$. As of March 2021, there are 6059 (3\%) confirmed cases, with $4092(67.5 \%)$ recoveries and $185(3 \%)$ deaths among the 202,214 persons residing in Garmian region ${ }^{[24]}$.

This KAP study was conducted to assess adults' knowledge, attitudes, and practice toward the COVID-19 pandemic in Garmian region and the relationships among sociodemographic data and knowledge, attitudes, and practice, also to answer the following questions:

- Are there good levels of knowledge about COVID-19 in Garmian, positive attitudes about the control of the virus, and healthy practices to avoid the spread of the virus and to prevent getting infected?

- Are there differences in levels of knowledge, attitudes, and practice with different socio-demographic characteristics?

Is the level of education about COVID-19 affects the positive attitudes andlor the practice of healthy measures to prevent the spread of the virus?

\section{Methodology}

\section{Study design and setting:}

A quantitative, descriptive, online cross-sectional survey was conducted during a complete lockdown from 5th to 20th of April 2020 in Garmian, an administrative region in South-East of KRI consisting of three districts Khanaqin, Kifri and Kalar, all bordering Iran. It was not feasible to draw a community-based sample because of the lockdown measures imposed by the authorities. Data were collected online using a Google platform designed by the authors.

\section{Study participants and data collection}

A non-probability, convenient sampling was used for data collection. Participation was restricted to Garmian residents, males and females, age 18 years old and older who are willing to participate and have access to the internet. Questionnaires in Arabic and Kurdish languages were prepared and sent to public websites and community groups in the Garmian Region. Data were collected over a two-week period. Three hundred twentyfive participations have completed the survey.

\section{Study Questionnaire}

The questionnaire was based on a study conducted in Wuhan, China ${ }^{[16]}$. It was modified/adapted by the authors according to the study objectives and context. It consisted of two parts: demographics and KAP indicators. Demographic variables included age, gender, marital status, education, occupation, and current residence. Nine extra questions were added to the original questionnaire: two questions for knowledge, two for attitudes and five for practices. The final version consisted of 14 questions about knowledge, four questions about attitudes, and seven questions about practice with (yes, no, I do not know) answer options. The scores were as follow for knowledge $(0-4=$ poor knowledge; 5-9= moderate knowledge; 10-14= good knowledge); for attitudes ( $0-1=$ negative attitudes; $2=$ neutral attitudes; $3-4=$ positive attitudes $)$; and for practice $(0-2=$ poor practice; $3-4=$ fair practice; 5-7= good practice). The questionnaire was translated from English to Kurdish and Arabic; both versions were back translated and approved by linguistic experts. A correct answer was assigned one point and an incorrect/unknown answer was assigned 0 points. The total knowledge score ranged from 0 to 14 , with a higher score indicating a better knowledge of COVID-19. With respect to attitudes and practice, correct answers (yes) indicated positive attitudes and correct practice. The Cronbach's alpha coefficient of the questionnaire was 0.73 in our sample, indicating acceptable internal consistency.

\section{Ethical consideration}

The Ethical Committee of Research Center, University of Garmian reviewed and approved the study proposal and procedures before the survey was implemented on April $1^{\text {st }} 2020$ (GRCEC104). Informed consent was taken from the participants by clarifying the purpose of the study, giving a brief background about the study and by choosing "Agree to participate" prior to answering the questionnaire.

\section{Analysis Methods}

In this study the authors have employed a primarily univariate and multivariable regression data analyses. The univariate analysis was done for tabulating the frequency of social and demographic statistics. Due to the continuous scores that were found in the results, authors have done a multivariable linear regression analysis to identify factors related to knowledge, attitudes, and practice. All analyses were conducted using SPSS 22 (SPSS Inc., Chicago, Ill., USA).

\section{Results}

Social and Demographic Characteristics: 
In this study 325 participants have completed the online questionnaire; 138 (42.5\%) women and 187 (57.5\%) men. In Table 1 the social and demographic characteristics of the participants are showed. The mean of COVID-19 knowledge, Attitudes, and Practice scores were $7.14(\mathrm{SD}=2.89$, range: $1-14)$; $6.82(\mathrm{SD}=2.16$, range:4.12); $3.72(\mathrm{SD}=1.96$, range: $0-7)$ respectively.

Table 1: Social and demographic characteristics of the study participants

\begin{tabular}{|c|c|c|c|c|c|c|}
\hline Variable & Mean & SD & Min & Max & $\mathrm{N}$ & $\%$ \\
\hline Knowledge Score & 7.14 & 2.89 & 1 & 14 & & \\
\hline Attitude score & 6.82 & 2.16 & 4 & 12 & & \\
\hline Practice score & 3.72 & 1.96 & 0 & 7 & & \\
\hline \multicolumn{7}{|l|}{ Gender } \\
\hline Male & & & & & 187 & 57.5 \\
\hline Female & & & & & 138 & 42.5 \\
\hline \multicolumn{7}{|l|}{ Age } \\
\hline $18-29$ & & & & & 117 & 36.0 \\
\hline $30-39$ & & & & & 118 & 36.3 \\
\hline $40-49$ & & & & & 73 & 22.5 \\
\hline $50-59$ & & & & & 15 & 4.6 \\
\hline$>60$ & & & & & 2 & 0.6 \\
\hline \multicolumn{7}{|l|}{ Marital status } \\
\hline Married & & & & & 24 & 75.1 \\
\hline Single & & & & & 73 & 22.5 \\
\hline Widow\& divorced & & & & & 8 & 2.5 \\
\hline \multicolumn{7}{|l|}{ Education } \\
\hline Illiterate & & & & & 41 & 12.6 \\
\hline Read\& write & & & & & 36 & 11.1 \\
\hline
\end{tabular}

\begin{tabular}{|c|c|c|c|c|c|c|}
\hline Variable & Mean & SD & Min & Max & $\mathrm{N}$ & $\%$ \\
\hline Primary & & & & & 41 & 12.6 \\
\hline Secondary & & & & & 38 & 11.7 \\
\hline Preparatory & & & & & 16 & 4.9 \\
\hline Diploma & & & & & 45 & 13.8 \\
\hline Bachelor & & & & & 52 & 16.0 \\
\hline High diploma & & & & & 11 & 3.4 \\
\hline Master & & & & & 29 & 8.9 \\
\hline Ph.D. & & & & & 16 & 4.9 \\
\hline Occupation & & & & & & \\
\hline Governmental & & & & & 118 & 36.3 \\
\hline $\begin{array}{l}\text { Non- } \\
\text { governmental }\end{array}$ & & & & & 14 & 4.3 \\
\hline Retired & & & & & 2 & 0.6 \\
\hline $\begin{array}{l}\text { Jobless } \\
\text { (unemployed) }\end{array}$ & & & & & 30 & 9.2 \\
\hline Self-employed & & & & & 32 & 9.8 \\
\hline Housekeeper & & & & & 60 & 18.5 \\
\hline Student & & & & & 69 & 21.2 \\
\hline
\end{tabular}

The age of the majority of this study samples $(72.3 \%)$ were between the ages of 18 and 39; about 244 were married $(75.1 \%)$ and 73 were unmarried $(22.5 \%)$, while the others were widowed or divorced (2.5\%). The educations of the sample were as follow: $(13.8 \%)$ hold diploma and $33.2 \%$ of participants had a college or university degree. In terms of occupation, $118(36.3 \%)$ were government employees. Only $2(0.6 \%)$ of the participants were retired, while $30(9.2 \%)$ were unemployed.

Table 2: Responses about COVID-19 knowledge, attitudes, and practice among respondents:

\begin{tabular}{|c|c|c|c|c|}
\hline \multirow[b]{2}{*}{ S.no } & \multirow[b]{2}{*}{ Questions } & \multicolumn{3}{|c|}{ Frequency } \\
\hline & & Correct $(\mathrm{N}, \%)$ & $\begin{array}{c}\text { Incorrect }(\mathrm{N}, \\
\%)\end{array}$ & $\begin{array}{l}\text { I don't know } \\
(\mathrm{N}, \%)\end{array}$ \\
\hline K1 & $\begin{array}{l}\text { To prevent COVID-19 infection, should individuals avoid going to } \\
\text { crowded places such as bus stations and avoid taking public } \\
\text { transportations? }\end{array}$ & $242(74.4)$ & $9(2.8)$ & $74(22.7)$ \\
\hline $\mathrm{K} 2$ & To avoid transmitting of COVID-19, should we stop shaking hands? & $219(67.5)$ & $19(5.8)$ & $87(26.7)$ \\
\hline K3 & $\begin{array}{l}\text { Do you think that COVID-19 virus spreads via respiratory droplets } \\
\text { from infected individuals? }\end{array}$ & 201(61.7) & $40(12.2)$ & $84(26.2)$ \\
\hline K4 & $\begin{array}{l}\text { Do you think fear and anxiety of getting infected with COVID-19 } \\
\text { decreases immunity? }\end{array}$ & $127(38.9)$ & $58(17.8)$ & $140(43.2)$ \\
\hline K5 & $\begin{array}{l}\text { Do infected persons with COVID-2019 transmit the virus to others } \\
\text { when not feverish? }\end{array}$ & $116(35.6)$ & 128(39.2) & $81(25.1)$ \\
\hline K6 & Is there any effective cure for COVID-2019 at this time? & $124(38.06)$ & 136(41.7) & $65(19.9)$ \\
\hline K7 & $\begin{array}{l}\text { Are the principal clinical symptoms of COVID-19 fever, fatigue, dry } \\
\text { cough, and myalgia? }\end{array}$ & $204(62.6)$ & $49(14.9)$ & $73(22.5)$ \\
\hline K8 & $\begin{array}{l}\text { Are a stuffy - runny nose, and sneezing less common in persons } \\
\text { infected with the COVID-19 compared with the common cold? }\end{array}$ & 190(58.3) & $59(18.2)$ & $76(23.4)$ \\
\hline K9 & $\begin{array}{l}\text { Not all persons with COVID- } 2019 \text { will become severe cases. Do those } \\
\text { who are elderly and have chronic illnesses more likely to develop } \\
\text { severe cases? }\end{array}$ & $168(51.7)$ & $49(15.1)$ & $108(33.2)$ \\
\hline K10 & $\begin{array}{l}\text { Should ordinary residents wear masks and gloves to prevent infection } \\
\text { by the COVID-19 virus? }\end{array}$ & $158(48.5)$ & $78(24.1)$ & $89(27.4)$ \\
\hline K11 & $\begin{array}{l}\text { Is it necessary for children and young adults to take preventive } \\
\text { measures to prevent COVID-19 infection? }\end{array}$ & $131(40.2)$ & $81(24.9)$ & $113(34.8)$ \\
\hline K12 & $\begin{array}{l}\text { Is it necessary to isolate people who are infected with COVID-19 to } \\
\text { reduce the spread of the virus? }\end{array}$ & $188(57.7)$ & $98(30.0)$ & $39(12.3)$ \\
\hline
\end{tabular}




\begin{tabular}{l|l|l|l|l}
\hline K13 & $\begin{array}{l}\text { Do people who have contact with someone infected with a confirmed } \\
\text { case of COVID-19 be immediately isolated in a proper place for 14 } \\
\text { days? }\end{array}$ & $85(26.0)$ & $62(19.1)$ & $\mathbf{1 7 8 ( 5 4 . 8 )}$ \\
\hline K14 & $\begin{array}{l}\text { Is supportive treatment and early diagnosis of COVID-19 patients } \\
\text { helpful in curing a case? }\end{array}$ & $\mathbf{1 5 6 ( 4 7 . 8 )}$ & $45(13.8)$ & $124(38.3)$ \\
\hline A1 & $\begin{array}{l}\text { Do you think that Garmian authorities have been taking the necessary } \\
\text { steps to control the COVID-19 pandemic? }\end{array}$ & $129(39.7)$ & $\mathbf{1 4 2 ( 4 3 . 7 )}$ & $54(16.6)$ \\
\hline A2 & $\begin{array}{l}\text { Do you think that people in Garmian region are following the public } \\
\text { health instructions properly to protect themselves from COVID-19? }\end{array}$ & $65(20.0)$ & $\mathbf{1 9 2 ( 5 9 . 1 )}$ & $68(20.9)$ \\
\hline A3 & $\begin{array}{l}\text { Do you agree that the COVID-19 pandemic will finally be successfully } \\
\text { controlled? }\end{array}$ & $\mathbf{1 5 0}(\mathbf{4 6 . 0 5 )}$ & $112(34.3)$ & $63(19.5)$ \\
\hline A4 & $\begin{array}{l}\text { Do you think that following the protective instructions could help in } \\
\text { controlling COVID-19? }\end{array}$ & $\mathbf{1 6 6 ( 5 0 . 9 )}$ & $93(29.5)$ & $63(19.5)$ \\
\hline P1 & $\begin{array}{l}\text { In recent days, have you gone to any crowded place such as } \\
\text { markets/public places? }\end{array}$ & $\mathbf{1 8 6 ( 5 7 . 1 )}$ & $62(19.1)$ & $77(23.7)$ \\
\hline P2 & In recent days, have you visited your friends, relatives? & $\mathbf{1 8 7 ( 5 7 . 4 )}$ & $49(15.1)$ & $61(27.4)$ \\
\hline P3 & Do you respect the curfew? & $\mathbf{1 8 3 ( 6 5 . 1 )}$ & $78(24.1)$ & $64(19.7)$ \\
\hline P4 & In recent days, have you worn a mask when leaving home? & $\mathbf{1 4 4 ( 4 7 . 4 )}$ & $99(30.3)$ & $72(22.2)$ \\
\hline P5 & In recent days, do you wear gloves when you go out? & $\mathbf{1 4 1 ( 4 3 . 2 )}$ & $105(32.4)$ & $79(24.3)$ \\
\hline P6 & $\begin{array}{l}\text { In recent days, do you usually wash your hands when entering your } \\
\text { home? }\end{array}$ & $\mathbf{1 9 3 ( 5 9 . 2 )}$ & $69(21.1)$ & $63(19.3)$ \\
\hline P7 & $\begin{array}{l}\text { If you develop COVID-19 signs and symptoms, are you ready to report } \\
\text { your symptoms to the authorities? }\end{array}$ & $\mathbf{1 6 9 ( 5 1 . 8 )}$ & $72(22.10)$ & $84(25.7)$ \\
\hline
\end{tabular}

Level of knowledge, attitudes, and practice were also assessed across the education groups. Figures $1-3$ show the results. Figure 1 suggests that the level of knowledge increases with educational attainment, Figure 2 shows that there were no discernible patterns across education groups although higher education levels decreased negative attitude, and Figure 3 indicate that with higher educational levels correct practices toward COVID-19 were higher.

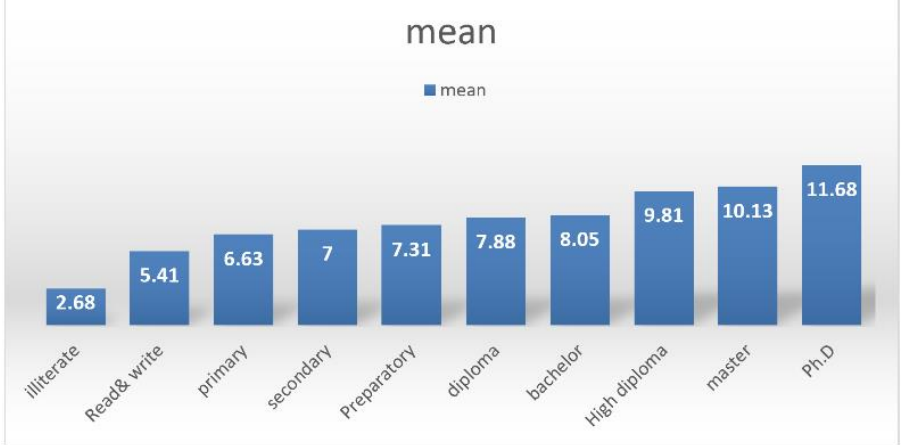

Figure 1: Knowledge levels about COVID-19 by education groups

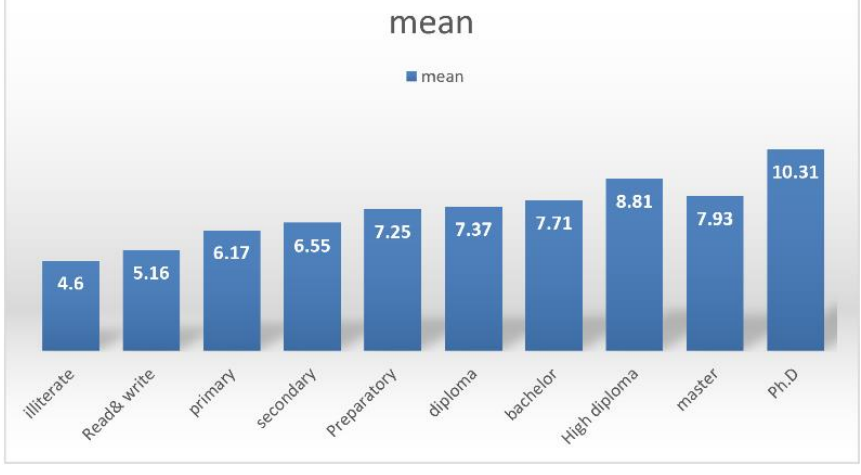

Figure 2: Attitude about COVID-19 by level of educational groups

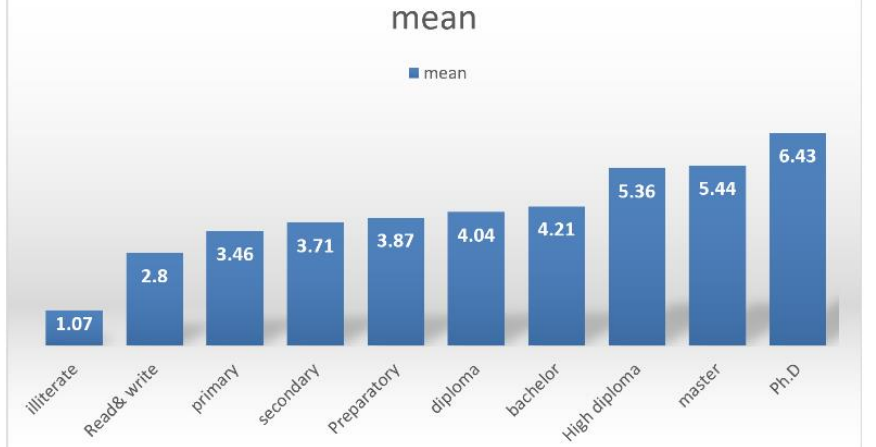

Figure 3: Correct practices for COVID-19 by levels of education

Table 3: Correlations among knowledge, attitude and practice:

\begin{tabular}{|l|l|c|c|c|}
\hline \multicolumn{2}{|l|}{} & Knowledge & Attitude & Practice \\
\hline Knowledge & *r $_{\text {(p-value })}$ & 1 & $0.19(0.001)^{* *}$ & $0.26(0.000)^{* *}$ \\
\hline Attitude & $* \mathrm{r}$ (p-value) & & 1 & $0.000)^{* *}$ \\
\hline
\end{tabular}

*. $\mathrm{r}$ correlation coefficient. **. Correlation is significant (0.01). This table certifies significant correlation among knowledge, attitude and practice. 
Table 4: Association between participants' characteristics and level of knowledge

\begin{tabular}{|c|c|c|c|c|c|}
\hline \multirow{2}{*}{\multicolumn{2}{|c|}{ Age Groups }} & \multicolumn{3}{|c|}{ Knowledge Levels } & \multirow{3}{*}{$\begin{array}{c}\text { Total } \\
7\end{array}$} \\
\hline & & \multirow{2}{*}{$\begin{array}{c}\text { Good } \\
3\end{array}$} & \multirow{2}{*}{$\begin{array}{c}\text { Moderate } \\
3\end{array}$} & \multirow{2}{*}{$\begin{array}{c}\text { Poor } \\
1\end{array}$} & \\
\hline$<2$ & $\mathrm{~F}$ & & & & \\
\hline$<20$ & $\%$ & $42.9 \%$ & $42.9 \%$ & $14.3 \%$ & $100 \%$ \\
\hline \multirow{2}{*}{$20-50$} & $\mathrm{~F}$ & 154 & 137 & 7 & 298 \\
\hline & $\%$ & $51.7 \%$ & $46.0 \%$ & $2.3 \%$ & $100 \%$ \\
\hline \multirow{3}{*}{$>50$} & $\mathrm{~F}$ & 13 & 6 & 1 & 20 \\
\hline & $\%$ & $65.0 \%$ & $30.0 \%$ & $5.0 \%$ & $100 \%$ \\
\hline & & Chi-Square & 5.73 & $\mathbf{P}$ & 0.22 \\
\hline \multicolumn{6}{|l|}{ Gender } \\
\hline \multirow[t]{2}{*}{ Male } & $\mathrm{F}$ & 95 & 74 & 6 & 175 \\
\hline & $\%$ & $54.3 \%$ & $42.3 \%$ & $3.4 \%$ & $100.0 \%$ \\
\hline \multirow[t]{3}{*}{ Female } & $\mathrm{F}$ & 75 & 72 & 3 & 150 \\
\hline & $\%$ & $50.0 \%$ & $48.0 \%$ & $2.0 \%$ & $100.0 \%$ \\
\hline & & Chi-Square & 1.47 & $\mathbf{P}$ & 0.48 \\
\hline \multicolumn{6}{|l|}{ Marital Status } \\
\hline \multirow[t]{2}{*}{ Single } & $\mathrm{F}$ & 60 & 57 & 3 & 120 \\
\hline & $\%$ & $50.0 \%$ & $47.5 \%$ & $2.5 \%$ & $100.0 \%$ \\
\hline \multirow[t]{3}{*}{ Married } & $\mathrm{F}$ & 110 & 89 & 6 & 205 \\
\hline & $\%$ & $53.7 \%$ & $43.4 \%$ & $2.9 \%$ & $100.0 \%$ \\
\hline & & Chi-Square & $\mathbf{0 . 5 3}$ & $\mathbf{P}$ & 0.78 \\
\hline \multicolumn{6}{|l|}{ Educations } \\
\hline \multirow[t]{2}{*}{ Postgraduate } & $\mathrm{F}$ & 57 & 42 & 2 & 101 \\
\hline & $\%$ & $56.4 \%$ & $41.6 \%$ & $2.0 \%$ & $100 \%$ \\
\hline \multirow[t]{2}{*}{ Institute/University } & $\mathrm{F}$ & 100 & 79 & 2 & 181 \\
\hline & $\%$ & $55.2 \%$ & $43.6 \%$ & $1.1 \%$ & $100 \%$ \\
\hline \multirow[t]{3}{*}{ Less than Institute } & $\mathrm{F}$ & 13 & 25 & 5 & 43 \\
\hline & $\%$ & $30.2 \%$ & $58.1 \%$ & $11.6 \%$ & $100 \%$ \\
\hline & & Chi-Square & 20.85 & $\mathbf{P}$ & 0.000 \\
\hline \multicolumn{6}{|l|}{ Occupations } \\
\hline \multirow[t]{2}{*}{ Gov’t. Employee } & $\mathrm{F}$ & 112 & 90 & 4 & 206 \\
\hline & $\%$ & $54.4 \%$ & $43.7 \%$ & $1.9 \%$ & $100.0 \%$ \\
\hline \multirow[t]{2}{*}{ Retired/Jobless } & $\mathrm{F}$ & 14 & 17 & 3 & 34 \\
\hline & $\%$ & $41.2 \%$ & $50.0 \%$ & $8.8 \%$ & $100.0 \%$ \\
\hline \multirow[t]{2}{*}{ Self-Employed } & $\mathrm{F}$ & 13 & 8 & 1 & 22 \\
\hline & $\%$ & $59.1 \%$ & $36.4 \%$ & $4.5 \%$ & $100.0 \%$ \\
\hline \multirow[t]{3}{*}{ Students } & $\mathrm{F}$ & 31 & 31 & 1 & 63 \\
\hline & $\%$ & $49.2 \%$ & $49.2 \%$ & $1.6 \%$ & $100.0 \%$ \\
\hline & & Chi-Square & 7.74 & $\mathbf{P}$ & 0.25 \\
\hline \multirow{2}{*}{ Total } & $\mathrm{F}$ & 170 & 146 & 9 & 325 \\
\hline & $\%$ & $52.3 \%$ & $44.9 \%$ & $2.8 \%$ & $100 \%$ \\
\hline
\end{tabular}

Table 5: Association between participants' characteristics and level of attitude

\begin{tabular}{|c|c|c|c|c|c|}
\hline \multirow{2}{*}{ Age Groups } & & Attitude Levels & & & \multirow{2}{*}{ Total } \\
\hline & & Positive & Neutral & Negative & \\
\hline \multirow{2}{*}{$<20$} & $\mathrm{~F}$ & 1 & 3 & 3 & 7 \\
\hline & $\%$ & $14.3 \%$ & $42.9 \%$ & $42.9 \%$ & $100.0 \%$ \\
\hline \multirow{2}{*}{$20-50$} & $\mathrm{~F}$ & 67 & 108 & 123 & 298 \\
\hline & $\%$ & $22.5 \%$ & $36.2 \%$ & $41.3 \%$ & $100.0 \%$ \\
\hline \multirow{3}{*}{$>50$} & $\mathrm{~F}$ & 4 & 10 & 6 & 20 \\
\hline & $\%$ & $20.0 \%$ & $50.0 \%$ & $30.0 \%$ & $100.0 \%$ \\
\hline & & Chi-Square & 1.86 & $\mathbf{P}$ & 0.76 \\
\hline \multicolumn{6}{|l|}{ Gender } \\
\hline Male & $\mathrm{F}$ & 38 & 64 & 73 & 175 \\
\hline
\end{tabular}




\begin{tabular}{|c|c|c|c|c|c|}
\hline & $\%$ & $21.7 \%$ & $36.6 \%$ & $41.7 \%$ & $100.0 \%$ \\
\hline \multirow{3}{*}{ Female } & $\mathrm{F}$ & 34 & 57 & 59 & 150 \\
\hline & $\%$ & $22.7 \%$ & $38.0 \%$ & $39.3 \%$ & $100.0 \%$ \\
\hline & & Chi-Square & 0.19 & $\mathbf{P}$ & 0.91 \\
\hline \multicolumn{6}{|l|}{ Marital Status } \\
\hline \multirow{2}{*}{ Single } & $\mathrm{F}$ & 28 & 41 & 51 & 120 \\
\hline & $\%$ & $23.3 \%$ & $34.2 \%$ & $42.5 \%$ & $100.0 \%$ \\
\hline \multirow[t]{3}{*}{ Married } & $\mathrm{F}$ & 44 & 80 & 81 & 205 \\
\hline & $\%$ & $21.5 \%$ & $39.0 \%$ & $39.5 \%$ & $100.0 \%$ \\
\hline & & Chi-Square & 0.77 & $\mathbf{P}$ & 0.68 \\
\hline \multicolumn{6}{|l|}{ Educations } \\
\hline \multirow[t]{2}{*}{ Postgraduate } & $\mathrm{F}$ & 17 & 44 & 40 & 101 \\
\hline & $\%$ & $16.8 \%$ & $43.6 \%$ & $39.6 \%$ & $100.0 \%$ \\
\hline \multirow[t]{2}{*}{ Institute/University } & $\mathrm{F}$ & 47 & 66 & 68 & 181 \\
\hline & $\%$ & $26.0 \%$ & $36.5 \%$ & $37.6 \%$ & $100.0 \%$ \\
\hline \multirow[t]{3}{*}{ Less than Institute } & $\mathrm{F}$ & 8 & 11 & 24 & 43 \\
\hline & $\%$ & $18.6 \%$ & $25.6 \%$ & $55.8 \%$ & $100.0 \%$ \\
\hline & & Chi-Square & 8.3 & $\mathbf{P}$ & 0.08 \\
\hline \multicolumn{6}{|l|}{ Occupations } \\
\hline \multirow[t]{2}{*}{ Gov Emil } & $\mathrm{F}$ & 46 & 80 & 80 & 206 \\
\hline & $\%$ & $22.3 \%$ & $38.8 \%$ & $38.8 \%$ & $100.0 \%$ \\
\hline \multirow[t]{2}{*}{ Retired/Jobless } & $\mathrm{F}$ & 11 & 7 & 16 & 34 \\
\hline & $\%$ & $32.4 \%$ & $20.6 \%$ & $47.1 \%$ & $100.0 \%$ \\
\hline \multirow[t]{2}{*}{ Self-employ } & $\mathrm{F}$ & 5 & 8 & 9 & 22 \\
\hline & $\%$ & $22.7 \%$ & $36.4 \%$ & $40.9 \%$ & $100.0 \%$ \\
\hline \multirow[t]{3}{*}{ Students } & $\mathrm{F}$ & 10 & 26 & 27 & 63 \\
\hline & $\%$ & $15.9 \%$ & $41.3 \%$ & $42.9 \%$ & $100.0 \%$ \\
\hline & & Chi-Square & 6.23 & $\mathbf{P}$ & 0.39 \\
\hline \multirow{2}{*}{ Total } & $\mathrm{F}$ & 72 & 121 & 132 & 325 \\
\hline & $\%$ & $22.2 \%$ & $37.2 \%$ & $40.6 \%$ & $100.0 \%$ \\
\hline
\end{tabular}

Table 6: Association between participants' characteristics and level of practice

\begin{tabular}{|c|c|c|c|c|c|}
\hline \multirow{2}{*}{\multicolumn{2}{|c|}{ Age Groups }} & \multicolumn{3}{|c|}{ Practice Levels } & \multirow{3}{*}{$\begin{array}{c}\text { Total } \\
7\end{array}$} \\
\hline & & Good & Fair & Poor & \\
\hline \multirow{2}{*}{$<20$} & $\mathrm{~F}$ & 1 & 3 & 3 & \\
\hline & $\%$ & $14.3 \%$ & $42.9 \%$ & $42.9 \%$ & $100.0 \%$ \\
\hline \multirow[b]{2}{*}{$20-50$} & $\mathrm{~F}$ & 137 & 58 & 103 & 298 \\
\hline & $\%$ & $46.0 \%$ & $19.5 \%$ & $34.6 \%$ & $100.0 \%$ \\
\hline \multirow{3}{*}{$>50$} & $\mathrm{~F}$ & 10 & 4 & 6 & 20 \\
\hline & $\%$ & $50.0 \%$ & $20.0 \%$ & $30.0 \%$ & $100.0 \%$ \\
\hline & & Chi-Square & 7.32 & $\mathbf{P}$ & 0.44 \\
\hline \multicolumn{6}{|l|}{ Gender } \\
\hline \multirow[t]{2}{*}{ Male } & $\mathrm{F}$ & 66 & 37 & 72 & 175 \\
\hline & $\%$ & $37.7 \%$ & $21.1 \%$ & $41.1 \%$ & $100.0 \%$ \\
\hline \multirow[t]{3}{*}{ Female } & $\mathrm{F}$ & 82 & 28 & 40 & 150 \\
\hline & $\%$ & $54.7 \%$ & $18.7 \%$ & $26.7 \%$ & $100.0 \%$ \\
\hline & & Chi-Square & 10.26 & $\mathbf{P}$ & 0.006 \\
\hline \multicolumn{6}{|l|}{ Marital Status } \\
\hline \multirow[t]{2}{*}{ Single } & $\mathrm{F}$ & 57 & 18 & 45 & 120 \\
\hline & $\%$ & $47.5 \%$ & $15.0 \%$ & $37.5 \%$ & $100.0 \%$ \\
\hline \multirow[t]{3}{*}{ Married } & $\mathrm{F}$ & 91 & 47 & 67 & 205 \\
\hline & $\%$ & $44.4 \%$ & $22.9 \%$ & $32.7 \%$ & $100.0 \%$ \\
\hline & & Chi-Square & 3.01 & $\mathbf{P}$ & 0.22 \\
\hline \multicolumn{6}{|l|}{ Educations } \\
\hline \multirow[t]{2}{*}{ Postgraduate } & $\mathrm{F}$ & 46 & 23 & 32 & 101 \\
\hline & $\%$ & $45.5 \%$ & $22.8 \%$ & $31.7 \%$ & $100.0 \%$ \\
\hline
\end{tabular}




\begin{tabular}{|c|c|c|c|c|c|}
\hline Institute/University & $\mathrm{F}$ & 86 & 33 & 62 & 181 \\
\hline & $\%$ & $47.5 \%$ & $18.2 \%$ & $34.3 \%$ & $100.0 \%$ \\
\hline \multirow{3}{*}{ Less than Institute } & $\mathrm{F}$ & 16 & 9 & 18 & 43 \\
\hline & $\%$ & $37.2 \%$ & $20.9 \%$ & $41.9 \%$ & $100.0 \%$ \\
\hline & & Chi-Square & 2.41 & $\mathbf{P}$ & 0.66 \\
\hline \multicolumn{6}{|l|}{ Occupations } \\
\hline \multirow[t]{2}{*}{ Gov employ } & $\mathrm{F}$ & 102 & 39 & 65 & 206 \\
\hline & $\%$ & $49.5 \%$ & $18.9 \%$ & $31.6 \%$ & $100.0 \%$ \\
\hline \multirow[t]{2}{*}{ Retired/Jobless } & $\mathrm{F}$ & 13 & 8 & 13 & 34 \\
\hline & $\%$ & $38.2 \%$ & $23.5 \%$ & $38.2 \%$ & $100.0 \%$ \\
\hline \multirow[t]{2}{*}{ Self-employ } & $\mathrm{F}$ & 14 & 3 & 5 & 22 \\
\hline & $\%$ & $63.6 \%$ & $13.6 \%$ & $22.7 \%$ & $100.0 \%$ \\
\hline \multirow[t]{3}{*}{ Students } & $\mathrm{F}$ & 19 & 15 & 29 & 63 \\
\hline & $\%$ & $30.2 \%$ & $23.8 \%$ & $46.0 \%$ & $100.0 \%$ \\
\hline & & Chi-Square & 11.17 & $\mathbf{P}$ & 0.08 \\
\hline \multirow{2}{*}{ Total } & $\mathrm{F}$ & 148 & 65 & 112 & 325 \\
\hline & $\%$ & $45.5 \%$ & $20.0 \%$ & $34.5 \%$ & $100.0 \%$ \\
\hline
\end{tabular}

Tables 4, 5, and 6 show the relationship between sociodemographic characteristics with knowledge, attitudes, and practice respectively. The chi-square analysis indicates no significance association, only for educational level and knowledge (0.000), also for gender and practice (0.006) which both of them are highly significant.

Table 7: Comparison of social and demographic characteristics, and mean KAP scores:

\begin{tabular}{|c|c|c|c|c|c|c|c|c|c|c|c|}
\hline \multirow{2}{*}{ Variable } & \multirow[b]{2}{*}{$\mathrm{N}$} & \multirow[b]{2}{*}{$\%$} & \multicolumn{3}{|c|}{ Knowledge score } & \multicolumn{3}{|c|}{ Attitude score } & \multicolumn{3}{|c|}{ Practice score } \\
\hline & & & Mean & SD & $\bar{P}$ & Mean & SD & $P$ & Mean & SD & $\mathrm{P}$ \\
\hline \multicolumn{12}{|l|}{ Gender } \\
\hline Male & 187 & 57.5 & 7.65 & 2.70 & $<0.001$ & 7.18 & 2.14 & $<0.001$ & 3.89 & 1.89 & 0.07 \\
\hline Female & 138 & 42.5 & 6.49 & 2.99 & & 6.33 & 2.10 & & 3.50 & 2.04 & \\
\hline \multicolumn{12}{|l|}{ Age } \\
\hline $18-29$ & 117 & 36.0 & 7.68 & 2.85 & $<0.001$ & 7.01 & 2.29 & $<0.05$ & 4.13 & 1.99 & $<0.001$ \\
\hline $30-39$ & 118 & 36.3 & 7.61 & 2.84 & & 7.16 & 2.15 & & 3.85 & 1.88 & \\
\hline $40-49$ & 73 & 22.5 & 6.12 & 2.56 & & 6.24 & 1.92 & & 3.15 & 1.79 & \\
\hline $50-59$ & 15 & 4.6 & 4.66 & 2.63 & & 5.60 & 1.50 & & 2.53 & 2.29 & \\
\hline$>60$ & 2 & 0.6 & 4.00 & 0.01 & & 5.50 & 0.70 & & 2.00 & 0.01 & \\
\hline \multicolumn{12}{|l|}{ Marital status } \\
\hline Married & 244 & 75.1 & 6.72 & 2.86 & $<0.001$ & 6.52 & 2.0 & 0.23 & 3.65 & 2.07 & $<0.05$ \\
\hline Single & 73 & 22.5 & 4.62 & 4.59 & & 6.12 & 3.04 & & 2.00 & 2.50 & \\
\hline Widow\& divorced & 8 & 2.5 & 7.35 & 2.79 & & 6.93 & 2.17 & & 3.80 & 1.89 & \\
\hline \multicolumn{12}{|l|}{ Education } \\
\hline Illiterate & 41 & 12.6 & 2.68 & 0.75 & $<0.001$ & 4.60 & 0.58 & $<0.001$ & 1.37 & 0.84 & $<0.001$ \\
\hline Read\& write & 36 & 11.1 & 5.41 & 1.50 & & 5.16 & 1.32 & & 2.80 & 1.30 & \\
\hline Primary & 41 & 12.6 & 6.63 & 1.86 & & 6.17 & 1.37 & & 3.46 & 1.68 & \\
\hline Secondary & 38 & 11.7 & 7.00 & 1.64 & & 6.55 & 1.55 & & 3.71 & 1.73 & \\
\hline Preparatory & 16 & 4.9 & 7.31 & 1.66 & & 7.25 & 1.77 & & 3.87 & 1.74 & \\
\hline Diploma & 45 & 13.8 & 7.88 & 1.95 & & 7.37 & 1.77 & & 4.04 & 1.56 & \\
\hline Bachelor & 52 & 16.0 & 8.05 & 1.75 & & 7.71 & 1.90 & & 4.21 & 1.74 & \\
\hline High diploma & 11 & 3.4 & 9.81 & 1.66 & & 8.81 & 1.72 & & 5.36 & 1.02 & \\
\hline Master & 29 & 8.9 & 10.13 & 2.37 & & 7.93 & 2.28 & & 5.44 & 1.21 & \\
\hline Ph.D. & 16 & 4.9 & 11.68 & 3.07 & & 10.31 & 2.16 & & 6.43 & 0.72 & \\
\hline \multicolumn{12}{|l|}{ Occupation } \\
\hline governmental & 118 & 36.3 & 7.29 & 2.73 & $<0.001$ & 7.16 & 2.16 & $<0.05$ & 3.97 & 1.81 & $<0.001$ \\
\hline Non-governmental & 14 & 4.3 & 5.92 & 2.97 & & 6.85 & 2.21 & & 2.71 & 2.05 & \\
\hline Retired & 2 & 0.6 & 9.50 & 2.12 & & 7.50 & 0.70 & & 6.50 & 0.70 & \\
\hline Jobless & 30 & 9.2 & 5.73 & 3.19 & & 6.23 & 2.43 & & 2.86 & 2.04 & \\
\hline Personal-job & 32 & 9.8 & 6.78 & 3.03 & & 6.81 & 2.23 & & 3.50 & 2.07 & \\
\hline Home keeper & 60 & 18.5 & 6.11 & 2.82 & & 6.03 & 1.96 & & 3.31 & 2.15 & \\
\hline Student & 69 & 21.2 & 7.68 & 2.42 & & 7.15 & 2.0 & & 4.26 & 1.68 & \\
\hline
\end{tabular}


In Table 7, all the scores for KAP were statistically different for all age groups, education and occupation groups. However, there was no statistically significant difference in attitudes across marital status. Gender differences, in male and female practice, were not significantly different.

Beside the univariate and non-parametric analyses that were used in this study, authors have also performed regression analysis. Scores were logged for all variables, and interpreted using ordinary least squares (OLS). Increased scores imply increased knowledge of the pandemic, more positive attitude to embrace preventive practices and the adoption of proper protective practices. The results are shown in Table 8

Table 8: Regression results of KAP-related factors for COVID-19

\begin{tabular}{|c|c|c|c|}
\hline & \multicolumn{3}{|l|}{ Attitude } \\
\hline & Knowledge & Attitude & Practice \\
\hline Variable & $\beta(\mathrm{SE})$ & $\beta(\mathrm{SE})$ & $\beta(\mathrm{SE})$ \\
\hline \multicolumn{4}{|l|}{ Gender } \\
\hline Female & $\begin{array}{l}-0.19(0.31) \\
(0.001) * *\end{array}$ & $\begin{array}{l}-0.18(0.23) \\
(0.001) * *\end{array}$ & $\begin{array}{l}-0.08(0.22) \\
(0.13)\end{array}$ \\
\hline \multicolumn{4}{|l|}{ Age } \\
\hline \multirow[t]{2}{*}{$18-29$} & $-0.01(0.36)$ & $-0.03(0.27)$ & $\begin{array}{l}-0.06(0 . \\
25)\end{array}$ \\
\hline & $(0.85)$ & $(0.58)$ & $(0.26)$ \\
\hline \multirow[t]{2}{*}{$40-49$} & $-0.21(0.41)$ & $-0.17(0.31)$ & $\begin{array}{l}-0.15(0 . \\
28)\end{array}$ \\
\hline & $(0.001) * *$ & $(0.05) *$ & $(0.05) *$ \\
\hline \multirow[t]{2}{*}{$50-59$} & $-0.21(0.76)$ & $-0.15(0.58)$ & $\begin{array}{l}-0.14(0 . \\
52)\end{array}$ \\
\hline & $(0.001) * *$ & $(0.05) *$ & $(0.05) *$ \\
\hline \multirow[t]{2}{*}{$>60$} & $-0.09(1.97)$ & $-0.06(1.52)$ & $\begin{array}{l}-0.07(1 . \\
37)\end{array}$ \\
\hline & $(0.06)$ & $(0.27)$ & $(0.17)$ \\
\hline \multicolumn{4}{|l|}{ Marital status } \\
\hline Single & $\begin{array}{l}-0.53(1.46) \\
(0.05) * \\
-0.51(1.5)\end{array}$ & $\begin{array}{l}-0.42(1.13) \\
(0.05) * \\
-0.39(1.17)\end{array}$ & $\begin{array}{l}-0.43(1.0) \\
(0.05) * \\
-0.47(1.0)\end{array}$ \\
\hline Married & $(0.05) *$ & $(0.05) *$ & $(0.05) *$ \\
\hline \multirow{2}{*}{$\begin{array}{l}\text { Widow\& } \\
\text { divorced }\end{array}$} & $-0.38(1.1)$ & $-0.22(1.1)$ & $-0.36(0.81)$ \\
\hline & $(0.001) * *$ & $(0.001) * *$ & $(0.05) *$ \\
\hline \multicolumn{4}{|l|}{ Education } \\
\hline Illiterate & $\begin{array}{l}0.36(0.97) \\
(0.001) * *\end{array}$ & $\begin{array}{l}-0.28(0.90) \\
(0.05) *\end{array}$ & $\begin{array}{l}0.48(0.78) \\
(0.001) * *\end{array}$ \\
\hline Read and write & $\begin{array}{l}0.04(0.95) \\
(0.64)\end{array}$ & $\begin{array}{l}-0.19(0.88) \\
(0.12)\end{array}$ & $\begin{array}{l}0.18(0.77) \\
(0.13)\end{array}$ \\
\hline Primary & $\begin{array}{l}0.16(0.92) \\
(0.13)\end{array}$ & $\begin{array}{l}0.03(0.86) \\
(0.81)\end{array}$ & $\begin{array}{l}0.01(0.75) \\
(0.91)\end{array}$ \\
\hline Secondary & $\begin{array}{l}0.12(0.97) \\
(0.24)\end{array}$ & $\begin{array}{l}0.04(0.90) \\
(0.97)\end{array}$ & $\begin{array}{l}0.04(0.78) \\
(0.75)\end{array}$ \\
\hline Preparatory & $\begin{array}{l}0.09(1.0) \\
(0.20)\end{array}$ & $\begin{array}{l}0.04(0.97) \\
(0.64)\end{array}$ & $\begin{array}{l}0.02(0.84) \\
(0.76)\end{array}$ \\
\hline Diploma & $\begin{array}{l}0.14(0.94) \\
(0.18)\end{array}$ & $\begin{array}{l}0.06(0.88) \\
(0.61)\end{array}$ & $\begin{array}{l}0.04(0.77) \\
(0.74)\end{array}$ \\
\hline High diploma & $0.31(0.92)$ & $0.22(0.86)$ & $0.07(0.75)$ \\
\hline
\end{tabular}

\begin{tabular}{llll}
\hline & Attitude & & \\
\hline & Knowledge & Attitude & Practice \\
\hline Variable & $\beta(\mathrm{SE})$ & $\beta(\mathrm{SE})$ & $\beta(\mathrm{SE})$ \\
\hline \multirow{3}{*}{ Bach lore } & $(0.05) *$ & $(0.14)$ & $(0.08)$ \\
& $0.26(1.0)$ & $0.19(0.99)$ & $0.14(0.86)$ \\
Master & $(0.001) * *$ & $(0.05) * *$ & $(0.05) *$ \\
& $0.42(0.98)$ & $0.19(0.92)$ & $0.22(0.80)$ \\
Ph.D. & $(0.001) * *$ & $(0.11)$ & $(0.05) *$ \\
Occupation & $0.44(1.0)$ & $0.38(0.96)$ & $0.27(0.83)$ \\
Non- & $(0.001) * *$ & $(0.001) * *$ & $(0.05) *$ \\
governmental & & & \\
& $-0.14(0.78)$ & $0.02(0.62)$ & $-0.13(0.54)$ \\
Retired & $(0.05) *$ & $(0.60)$ & $(0.05) *$ \\
Jobless & $-0.04(1.9)$ & $-0.01(1.52)$ & $-0.10(1.3)$ \\
& $(0.42)$ & $(0.82)$ & $(0.05) *$ \\
Personal-job & $-0.03(0.42)$ & $0.002(0.32)$ & $-0.06(0.29)$ \\
& $(0.56)$ & $(0.97)$ & $(0.32) *$ \\
House keeper & $-0.11(0.55)$ & $0.04(0.42)$ & $0.07(0.38)$ \\
Student & $(0.05) *$ & $(0.40)$ & $(0.21)$ \\
Knowledge & $-0.24(0.44)$ & $-0.20(0.33)$ & $-0.13(0.30)$ \\
& $(0.001) *$ & $(0.001) * *$ & $(0.05) *$ \\
Standard error in parenthesis, P<0.001*, P<0.05** $)$ & $-0.16(0.39)$ \\
& $-0.25(0.56)$ & $-0.12(0.43)$ & $-0.05) *$ \\
& $(0.001) * *$ & $(0.05) *$ & $(0.05) *$ \\
& & $0.80(0.02)$ & $0.77(0.02)$ \\
& & & $(0.001) * *$ \\
\hline
\end{tabular}

As it is shown in Table 8, knowledge about COVID-19 of the age groups 18-28, 40-49, 50-59 and $\geq 60$, showed more knowledgeable about COVID-19 than the reference group (2939). Variables for the age groups $40-49(\beta=-0.21 ; \mathrm{p}<0.001)$, $50-59(\beta=-0.21 ; p<0.001)$ are statistically significant at the $1 \%$ level. In attitudes $40-49(\beta=-0.17 ; p<0.05)$ and $50-59(\beta=-$ $0.15 ; \mathrm{p}<0.05)$ are significantly different from the baseline. In case of practices for COVID-19, the age group 40-49 $(\beta=-0.17$; $\mathrm{p}<0.05)$ and 50-59 $(\beta=-0.14 ; \mathrm{p}<0.05)$ are associated with good practices. There was relationship between education and occupation with knowledge, attitude, and practice. The scores of KAP increased by increasing education level. Among occupation groups, the students have shown higher beta $=-25$.

Regarding gender, the results indicate that woman have higher knowledge $(\beta=-0.19 ; \mathrm{p}<0.001)$, positive attitudes $(\beta=-0.18$; $\mathrm{p}<0.001)$ compared to men. In practices for COVID-19 there were no significant difference between both genders. Also, no difference in KAP toward COVID-19 was observed in marital status of widow and married. While in marital status group, single participants had higher knowledge $(\beta=-0.53$; $\mathrm{p}<0.001)$, positive attitudes $(\beta=-0.42 ; \mathrm{p}<0.001)$, and good practices for COVID-19 $(\beta=-0.19 ; \mathrm{p}<0.001)$ compared to married participants.

For studying the statistical association between KAP scores, regression analysis was performed. Table 6 shows that every increase of $1 \%$ in knowledge score is associated an increase in a positive attitude and correct practices scores, of 0.80 and 0.77 , respectively. 
Table 9: chi-square test for gender and knowledge, attitudes, and practice:

\begin{tabular}{|c|c|c|c|}
\hline S.no & Questions & Pearson Chi-Square value & Sig. \\
\hline K1 & $\begin{array}{l}\text { To prevent COVID-19 infection, should individuals avoid going to crowded } \\
\text { places such as bus stations and avoid taking public transportations? }\end{array}$ & 2.924 & 0.087 \\
\hline $\mathrm{K} 2$ & To avoid transmitting of COVID-19, should we stop shaking hands? & 6.638 & $\mathbf{0 . 0 1 0}$ \\
\hline K3 & $\begin{array}{l}\text { Do you think that COVID-19 virus spreads via respiratory droplets from infected } \\
\text { individuals? }\end{array}$ & 0.372 & 0.542 \\
\hline K4 & $\begin{array}{l}\text { Do you think fear and anxiety of getting infected with COVID-19 decreases } \\
\text { immunity? }\end{array}$ & 3.172 & 0.075 \\
\hline K5 & $\begin{array}{l}\text { Do infected persons with COVID-2019 transmit the virus to others when not } \\
\text { feverish? }\end{array}$ & 14.612 & $\mathbf{0 . 0 0 0}$ \\
\hline K6 & Is there any effective cure for COVID-2019 at this time? & 13.678 & $\mathbf{0 . 0 0 0}$ \\
\hline K7 & $\begin{array}{l}\text { Are the principal clinical symptoms of COVID-19 fever, fatigue, dry cough, and } \\
\text { myalgia? }\end{array}$ & 1.215 & 0.27 \\
\hline K8 & $\begin{array}{l}\text { Are a stuffy - runny nose, and sneezing less common in persons infected with the } \\
\text { COVID-19 compared with the common cold? }\end{array}$ & 0.005 & 0.942 \\
\hline K9 & $\begin{array}{l}\text { Not all persons with COVID-2019 will become severe cases. Do those who are } \\
\text { elderly and have chronic illnesses more likely to develop severe cases? }\end{array}$ & 0.863 & 0.353 \\
\hline K10 & $\begin{array}{l}\text { Should ordinary residents wear masks and gloves to prevent infection by the } \\
\text { COVID-19 virus? }\end{array}$ & 8.059 & 0.005 \\
\hline K11 & $\begin{array}{l}\text { Is it necessary for children and young adults to take preventive measures to } \\
\text { prevent COVID-19 infection? }\end{array}$ & 0.247 & 0.619 \\
\hline K12 & $\begin{array}{l}\text { Is it necessary to isolate people who are infected with COVID-19 to reduce the } \\
\text { spread of the virus? }\end{array}$ & 0.439 & 0.538 \\
\hline K13 & $\begin{array}{l}\text { Do people who have contact with someone infected with a confirmed case of } \\
\text { COVID-19 be immediately isolated in a proper place for } 14 \text { days? }\end{array}$ & 0.022 & 0.881 \\
\hline K14 & $\begin{array}{l}\text { Is supportive treatment and early diagnosis of COVID-19 patients helpful in } \\
\text { curing a case? }\end{array}$ & 0.357 & 0.550 \\
\hline A1 & $\begin{array}{l}\text { Do you think that Garmian authorities have been taking the necessary steps to } \\
\text { control the COVID-19 pandemic? }\end{array}$ & 2.650 & 0.266 \\
\hline $\mathrm{A} 2$ & $\begin{array}{l}\text { Do you think that people in Garmian region are following the public health } \\
\text { instructions properly to protect themselves from COVID-19? }\end{array}$ & 5.578 & 0.061 \\
\hline A3 & $\begin{array}{l}\text { Do you agree that the COVID-19 pandemic will finally be successfully } \\
\text { controlled? }\end{array}$ & 2.115 & 0.347 \\
\hline A4 & $\begin{array}{l}\text { Do you think that following the protective instructions could help in controlling } \\
\text { COVID-19? }\end{array}$ & 6.649 & $\mathbf{0 . 0 3 9}$ \\
\hline $\mathrm{P} 1$ & $\begin{array}{l}\text { In recent days, have you gone to any crowded place such as markets/public } \\
\text { places? }\end{array}$ & 2.879 & 0.090 \\
\hline $\mathrm{P} 2$ & In recent days, have you visited your friends, relatives? & 26.973 & 0.000 \\
\hline $\mathrm{P} 3$ & Do you respect the curfew? & 0.675 & 0.411 \\
\hline $\mathrm{P} 4$ & In recent days, have you worn a mask when leaving home? & 5.214 & $\mathbf{0 . 0 2 2}$ \\
\hline P5 & In recent days, do you wear gloves when you go out? & 0.546 & 0.460 \\
\hline P6 & In recent days, do you usually wash your hands when entering your home? & 0.002 & 0.967 \\
\hline P7 & $\begin{array}{l}\text { If you develop COVID-19 signs and symptoms, are you ready to report your } \\
\text { symptoms to the authorities? }\end{array}$ & 1.288 & 0.256 \\
\hline
\end{tabular}

The Chi-square analysis shows that there is no significance relationship between the gender and knowledge, attitudes, and practice, only in few questions as follows: $\mathrm{K} 2, \mathrm{~K} 5, \mathrm{~K} 6$, and $\mathrm{K} 10$; A4; and P2, P4 for knowledge, attitudes, and practice respectively.

\section{Discussions}

To the best of our knowledge, this is the first KAP study among this population in Garmian Region and the second in KRI during the COVID-19 pandemic. Participants had positive and negative attitudes with similar rates. Questions A1 and A2 had higher negative responses, while A3 and A4 had higher positive attitudes. The positive attitudes were that this pandemic will end and that applying protective measures will help to prevent the virus spread. The negative attitudes were about the administration's necessary steps in controlling the spread of the pandemic in the region, and that the population of the region is not following instructions properly. In spite of negative attitudes, the KRI authorities played a crucial role in controlling the spread of the virus. Studies show that the lockdown by governments was effective in controlling the spread of the COVID-19 ${ }^{[25,26]}$. 
While in the practice questions, all the seven questions (P1-P7) had higher rates of correct answers, which indicates good practice levels among participants.

The majority of the participants show a good level of knowledge about COVID-19. Studies, conducted in Iraq ${ }^{[7,8,9,10,27]}$, and in Iran, Syria and Saudi Arabia, show similar satisfactory levels of knowledge, attitudes and practice [14, 28, 29]. Another study by Zhong et al, 2020 reported higher levels of knowledge (90\%) among Chinese at the very beginning of the pandemic.

Based on the finding of this study, the absence of COVID-19 in the Garmian region was not due to a good knowledge, attitudes and practice of the community, but to the strict lockdown measures, such as imposing a curfew, closure of schools and universities, markets and prevention of social gathering, wearing masks and using of distinct, implemented be the local authorities. After easing these measures in early May 2020, cases were recorded and increased rapidly. It was reported that easing the lockdown measures in Garmian region contributed to the second spike of COVID-19 after a two-week period in which no cases were recorded ${ }^{[27]}$.

After six months of the virus spreading globally, people in Garmian had less knowledge than the Chinese population for example despite the availability of many resources including news channels, studies, social media and newspapers, whom have been giving awareness and up to date statistics every day. This suggests that the community was not taking the pandemic seriously and was not paying much attention to the information disseminated/circulated. At the beginning of the lockdown, people refused to follow instructions and the government was struggling to keep community members sheltering in place and follow the protective/ preventive measures. Low knowledge levels about K5 and K6 explains the rapid spread of the pandemic. Higher levels of education have shown higher knowledge and good practices regarding COVID-19, but not for attitudes. At the same time, with higher educational levels, negative attitudes were less when compared with respondents in lower educational levels. Similar results were found in other KAP studies that were conducted among health workers and dentists in China, Italy, Iran, Jordan and Iraq respectively ${ }^{[11,18,19,21,22]}$ and among other Iraqi communities ${ }^{[8]}$.

The sociodemographic characteristics, age, education, and occupation were not significantly different for KAP. Gender and marital status were statistically significant for knowledge and practice. Ghazi et al, also found that there is a significant relationship between the knowledge score and both gender and living state ${ }^{[9]}$.

All the age groups had good knowledge, but the age group of 2939 had less knowledge compared to others. Higher positive attitudes and better practices were found among age groups 4049 and 50-59. These findings are similar to the findings of another study which reported that younger age groups in the KRI have better knowledge and more positive attitudes, while older age groups have better practices ${ }^{[10]}$. This explains that older people pay more attention to the information about COVID-19, since risks are higher for older people ${ }^{[31,32,33]}$.

We have found that increased knowledge is associated with in an increase of attitudes and practice as well. There is a relationship between education and occupation as the scores increased with increased educational levels. There is a direct relationship between knowledge, attitudes and correct practice, suggesting that correct knowledge leads to positive attitudes and correct, appropriate practice ${ }^{[34]}$.

We recommend preparing and disseminating health awareness campaigns to the population about public health preparedness and preventive measures in dealing with disease outbreaks. In addition, we encourage researchers to conduct studies in the region to inform decision makers on best practices.

\section{Conclusion}

The assessment of knowledge, attitudes and practices of adults in the Garmian Region with respect to COVID-19 has shown that more than half of the study participants had good Knowledge and high levels of correct practice. There is a significant relationship between knowledge and attitudes and correct practice; participants with higher knowledge score have more positive attitudes and better practices. There are no significance differences between the socio-demographic characteristics and levels of knowledge, attitudes and practices. The findings of this study will inform the decision makers in the KRI and in Garmian region about the measure to put in place when facing future disease outbreaks and public health emergencies.

\section{Limitations}

The data of this study was collected online, and this excluded participants who did not have access to internet or whom are not familiar with such platform to participate. This makes it difficult for the results to be generalized. The study was conducted when there were only few cases in the region. Therefore, the results of this study might have been different if conducted among the general population and when cases and mortality increased dramatically.

\section{Acknowledgements}

The authors acknowledge the participants for their time and answering the questionnaire.

\section{Author's contribution}

MY designed the online form and performed the data collection; MA did the statistical analysis; NA, NA, and DJ worked on the writing of the research sections.

\section{Conflict of interests}

None.

\section{References}

1. Xiang YT, Yang Y, Li W, Zhang L, Zhang Q, Cheung T, Ng CH. Timely mental health care for the 2019 novel coronavirus outbreak is urgently needed. The Lancet Psychiatry. 2020 Mar 1;7(3):228-9.

2. World Health Organization (WHO). Rolling updates on coronavirus disease (COVID19). 2020. Date of access August 17th 2020. Available at https://www.who.int/emergencies/diseases/novel-coronavirus-2019/events-as-theyhappen

3. GOV.KRD. Corona Virus, COVID-19. 2020. Access date August $18^{\text {th }} 2020$. Available at https://gov.krd/coronavirus-en/situation-update/\#moh-monitoring 
4. Lobo D, Sams LM, Fernandez SL. Correlation between health professionals knowledge, attitude and practice about infection control measures. Journal of Medical \& Allied Sciences. 2019;9(1):26-31

5. Kaushal G, Doke P, Shah A, Verma V. An analysis of knowledge, attitude and practices regarding standard precautions of infection control and impact of knowledge and attitude of ICU nurses on self-reported practices of infection control. Int J Res Found Hosp Healthcare Admin. 2015 Jul;2:79-85

6. Maude RR, Jongdeepaisal M, Skuntaniyom S, Muntajit T, Blacksell SD, Khuenpetch W, Pan-Ngum W, Taleangkaphan K, Malathum K, Maude RJ. Improving knowledge, attitudes and practice to prevent COVID-19 transmission in healthcare workers and the public in Thailand. BMC Public Health. 2021 Dec;21(1):1-4.

7. Al-Shatari SA. Knowledge about Covid-19: Sample From Iraqi People. European Journal of Molecular \& Clinical Medicine. 2020 Dec 8;7(10):185-97.

8. Jadoo SA, Alhusseiny AH, Yaseen SM, Al-Samarrai MA, Al-Delaimy AK, Abed MW, Hassooni HR. Knowledge, attitude, and practice toward COVID-19 among Iraqi people: a web-based cross-sectional study. Journal of Ideas in Health. 2020 Dec 19;3(Special2):258-65.

9. Ghazi HF, Taher TM, AbdalQader MA, Raheema RH, Baobaid MF, Hasan TN Knowledge, Attitude, and Practice Regarding Coronavirus Disease-19: PopulationBased Study in Iraq. Open Access Macedonian Journal of Medical Sciences. 2020 Sep 20;8(T1):137-41.

10. Ali ZR, Sharif BO, Kamali AS, Abbas VT, Ahmed AK, Mahmood SO, Abdulqader SA. Community-Based Assessment of Knowledge, Attitudes, and Practices Towards COVID-19: an Epidemiological Survey in Kurdistan Region, Iraq. Kurdistan Journal of Applied Research. 2020 Oct 11:1-2.

11. Khalil NS, Al-Yuzbaki DB, Tawfeeq RS. COVID-19 knowledge, attitude and practice among medical undergraduate students in Baghdad City. EurAsian Journal of BioSciences. 2020 Oct 21;14(2):4179-86.

12. Hussein NR, Naqid IA, Jacksi K, Abdi BA. Assessment of knowledge, attitudes, and practices toward COVID-19 virus among university students in Kurdistan region, Iraq: Online cross-sectional study. Journal of family medicine and primary care. 2020 Sep;9(9):4809.

13. Rahim BI. Knowledge, Attitudes, and Practices of Staff and Students at Sulaimani Polytechnic University towards COVID-19/Iraq. Indian Journal of Forensic Medicine \& Toxicology. 2020 Oct; 14(4):815.

14. Al-Hanawi MK, Angawi K, Alshareef N, Qattan AM, Helmy HZ, Abudawood Y, Alqurashi M, Kattan WM, Kadasah NA, Chirwa GC, Alsharqi O. Knowledge, attitude and practice toward COVID-19 among the public in the Kingdom of Saudi Arabia: a cross-sectional study. Frontiers in Public Health. 2020;8.

15. Azlan AA, Hamzah MR, Sern TJ, Ayub SH, Mohamad E. Public knowledge, attitudes and practices towards COVID-19: A cross-sectional study in Malaysia. Plos one. 2020 May 21;15(5): e0233668.

16. Zhong BL, Luo W, Li HM, Zhang QQ, Liu XG, Li WT, Li Y. Knowledge, attitudes, and practices towards COVID-19 among Chinese residents during the rapid rise period of the COVID-19 outbreak: a quick online cross-sectional survey. International journal of biological sciences. 2020;16(10):1745.

17. Reuben RC, Danladi MM, Saleh DA, Ejembi PE. Knowledge, attitudes and practices towards COVID-19: an epidemiological survey in North-Central Nigeria. Journal of community health. 2020 Jul 7:1-4.

18. Alzoubi H, Alnawaiseh N, Al-Mnayyis A, Lubad MA, Aqel A, Al-Shagahin H. COVID-19-knowledge, attitude and practice among medical and non-medical University Students in Jordan. J Pure Appl Microbiol. 2020 Mar;14(1):17-24.

19. Taghrir MH, Borazjani R, Shiraly R. COVID-19 and Iranian medical students; a survey on their related-knowledge, preventive behaviors and risk perception. Arch Iran Med. 2020;23(4):249-254. doi:10.34172/aim.2020.06

20. Khader Y, Al Nsour M, Al-batayneh OB, et al. Dentists' awareness, perception, and attitude regarding COVID-19 and infection control: cross-sectional study among jordanian dentists. JMIR PUBLIC Heal Surveill. 2020; 6:2. doi:10.2196/18798
21. Zhang M, Zhou M, Tang F, et al. Knowledge, attitude and practice regarding COVID19 among health care workers in Henan, China. J Hosp Infect. 2020;105(2):183-187. doi: 10.1016/j.jhin.2020.04.012

22. Moro M, Pietro VG, Capraro M, et al. 2019-novel coronavirus survey: knowledge and attitudes of hospital staff of a large Italian teaching hospital. Acta Biomed. 2020;91(11):29-34. doi:10.23750/ abm. v91i3-S.9419

23. Sohrabi C, Alsafi Z, O'Neill N, Khan M, Kerwan A, Al-Jabir A, Iosifidis C, Agha R. World Health Organization declares global emergency: A review of the 2019 novel coronavirus (COVID-19). International journal of surgery. 2020 Apr 1;76:71-6.

24. Zhyan News. (2021). Latest statistics in Garmian region about COVID-19. Access date: $\quad$ March $5, \quad 2021 . \quad$ Retrieved from https://www.facebook.com/ZhyanMedia/videos/820253248527607

25. Lai S, Ruktanonchai NW, Zhou L, Prosper O, Luo W, Floyd JR, Wesolowski A, Santillana M, Zhang C, Du X, Yu H. Effect of non-pharmaceutical interventions to contain COVID-19 in China. Nature. 2020 Sep;585(7825):410-3.

26. Tian H, Liu Y, Li Y, Wu CH, Chen B, Kraemer MU, Li B, Cai J, Xu B, Yang Q, Wang B. An investigation of transmission control measures during the first 50 days of the COVID-19 epidemic in China. Science. 2020 May 8;368(6491):638-42.

27. Wadood MA, Lee LL, Huq MM, Mamun AS, Mohd S, Hossain MG. Practice and perception of Bangladeshi adults toward COVID-19: a cross-sectional study. 2020. Research Square, a preprint. file:///C:/Users/Lenovo/Downloads/v1\%20(1).pdf

28. Honarvar B, Lankarani KB, Kharmandar A, Shaygani F, Zahedroozgar M, Haghighi MR, Ghahramani S, Honarvar H, Daryabadi MM, Salavati Z, Hashemi SM. Knowledge, attitudes, risk perceptions, and practices of adults toward COVID-19: a population and field-based study from Iran. International journal of public health. 2020 Jul;65(6):731-9.

29. REACH. COVID-19 Knowledge, Attitudes and Practices (KAP) Survey: Northeast Syria Analysis - April (Rounds 1) and May (Round 2) 2020. 2020. Available at: https://reliefweb.int/report/syrian-arab-republic/covid-19-knowledge-attitudes-andpractices-kap-survey-northeast-syria-0

30. Ali KM, Tawfeeq HM, Rostam HM. COVID-19 Second Spike as an Aftermath of the Sudden Restrictions Ease: Kurdistan Region of Iraq as an Example. Passer Journal. 2020 Sep 1;2(2):57-61.

31. Shahid Z, Kalayanamitra R, McClafferty B, Kepko D, Ramgobin D, Patel R, Aggarwal CS, Vunnam R, Sahu N, Bhatt D, Jones K. COVID-19 and older adults: what we know. Journal of the American Geriatrics Society. 2020 May;68(5):926-9.

32. Nanda A, Vura NV, Gravenstein S. COVID-19 in older adults. Aging clinical and experimental research. $2020 \mathrm{Jul} ; 32: 1199-202$.

33. Yang Y, Li W, Zhang Q, Zhang L, Cheung T, Xiang YT. Mental health services for older adults in China during the COVID-19 outbreak. The Lancet Psychiatry. 2020 Apr 1;7(4):e19.

34. Puspitasari IM, Yusuf L, Sinuraya RK, Abdulah R, Koyama H. Knowledge, attitude, and practice during the COVID-19 pandemic: a review. Journal of Multidisciplinary Healthcare. 2020 Jul 30;13:727-33. 